Check for updates

Edinburgh

Cite this as: BMJ 2021;375:n3144 http://dx.doi.org/10.1136/bmj.n3144 Published: 23 December 2021

\title{
Covid-19: Early studies give hope omicron is milder than other variants
}

\section{Bryan Christie}

Optimism is growing that the increasingly dominant omicron variant of SARS-CoV-2 may produce less severe disease than previous strains, after the findings of three early studies into the outcome of infection with the variant.

Researchers in England, Scotland, and South Africa have found the risk of admission to hospital to be between $15 \%$ and $80 \%$ lower with omicron than the delta variant. The findings have not been peer reviewed, and all three studies accept limitations in the research, but the unanimity of the findings has been welcomed. "In my view, there is now solid reason to favour a more optimistic outcome of omicron in the UK than was feared," said James Naismith, director of the Rosalind Franklin Institute at the University of Oxford.

Scientists at Imperial College London estimated that people infected with omicron were 15-20\% less likely to attend hospital overall and 40-45\% less likely to be admitted for a night than people infected with the delta variant. ${ }^{1}$ The researchers' figures were based on an analysis carried out in the first two weeks of December involving 56000 cases of omicron and 269 ooo of delta.

The risk of hospital admission was found to be the same among people infected with omicron who had received only two doses of vaccine as among those infected with delta, emphasising the importance of the booster roll-out currently being carried out in the UK. People who were unvaccinated faced the greatest risk of hospital admission.

Neil Ferguson from Imperial College London said, "Our analysis shows evidence of a moderate reduction in the risk of hospitalisation. However, this appears to be offset by the reduced efficacy of vaccines against infection with the omicron variant. Given the high transmissibility of the omicron virus, there remains the potential for health services to face increasing demand if omicron cases continue to grow at the rate that has been seen in recent weeks."

The Scottish study used national surveillance data to compare omicron and delta infections from the date of the first report of an omicron case, 23 November, to 19 December. ${ }^{2}$ It estimated that, if omicron behaved the same as delta, it would result in 57 hospital cases. In fact, there were only 15. Although this study was based on a small number of cases and very few elderly people, it pointed to a $65 \%$ lower risk of hospital admission. The study's findings also indicated that a third booster vaccine reduced the risk of symptoms from omicron by $57 \%$.

Jim McMenamin, the national covid-19 incident director at Public Health Scotland, described the findings as a "qualified good news story."
In South Africa, which has longer experience with omicron, the latest research has found that people who were given a diagnosis of omicron infection in October and November were $80 \%$ less likely to be admitted to hospital than people with other variants. ${ }^{3}$

Cheryl Cohen of the country's National Institute for Communicable Diseases and one of the authors of the study, said, "Compellingly, together our data really suggest a positive story of a reduced severity of omicron compared to other variants."

This was further reinforced by surveillance data showing significantly lower numbers of hospital admissions and deaths in the current omicron driven wave of infections than in previous waves, even though case numbers were much higher, she added.

It is estimated that $60-70 \%$ of South Africa's population have had a previous SARS-CoV-2 infection, offering them some level of immunity, and Cohen said that the country's experience with omicron may not be the same as other countries with high vaccination levels but low levels of population immunity. Imperial College London estimated that more than half of people in England may have been infected before omicron, and rates of hospital admission may be strongly affected by infection induced immunity.

The findings of the two UK studies were seen as encouraging. Raghib Ali, senior clinical research associate at the Medical Research Council's Epidemiology Unit in Cambridge, said the data on risk of hospital admission can be used to estimate the likely peak of infection and total number of admissions in the coming weeks. "While further data are needed to confirm these studies, the worst case scenarios that were presented last week can safely be excluded," he said.

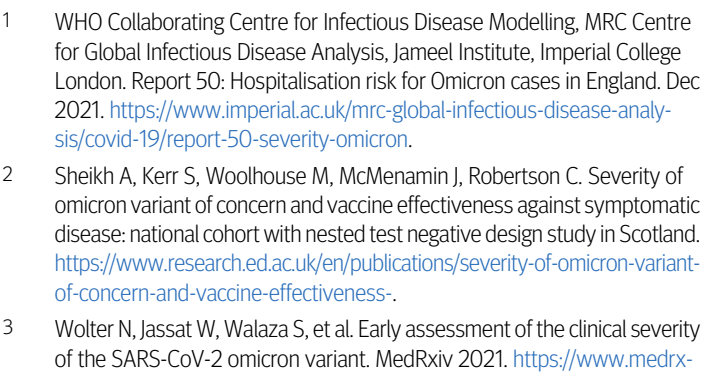

WHO Collaborating Centre for Infectious Disease Modelling, MRC Centre for Global Infectious Disease Analysis, Jameel Institute, Imperial College London. Report 50: Hospitalisation risk for Omicron cases in England. Dec 2021. https://www.imperial.ac.uk/mrc-global-infectious-disease-analysis/covid-19/report-50-severity-omicron.

2 Sheikh A, Kerr S, Woolhouse M, McMenamin J, Robertson C. Severity of omicron variant of concern and vaccine effectiveness against symptomatic disease: national cohort with nested test negative design study in Scotland https://www.research.ed.ac.uk/en/publications/severity-of-omicron-variantof-concern-and-vaccine-effectiveness-

3 Wolter N, Jassat W, Walaza S, et al. Early assessment of the clinical severity of the SARS-CoV-2 omicron variant. MedRxiv 2021. https://www. medrxiv.org/content/10.1101/2021.12.21.21268116v1.

This article is made freely available for use in accordance with BMJ's website terms and conditions for the duration of the covid-19 pandemic or until otherwise determined by BMJ. You may use, download and print the article for any lawful, non-commercial purpose (including text and data mining) provided that all copyright notices and trade marks are retained. 